\title{
AVALIAÇÃO DAS CONDIÇÕES DE PASTAGENS NO CERRADO BRASILEIRO POR MEIO DE GEOTECNOLOGIAS
}

\author{
Ricardo Guimarães Andrade ${ }^{1}$, Édson Luis Bolfe ${ }^{1}$, Daniel de Castro Victoria ${ }^{1}$, Sandra Furlan Nogueira $^{1}$
}

\begin{abstract}
RESUMO - O bioma Cerrado abrange 203,4 milhões de hectares (24\% do território nacional); desse total, cerca de 55 milhões de hectares são cultivados com pastagens. Este estudo objetivou avaliar as condições das pastagens cultivadas em municípios de abrangência do bioma Cerrado. Foram aplicadas técnicas de geoprocessamento e séries temporais de dados NDVI (Normalized Difference Vegetation Index) gerada a partir de imagens do sensor Spot-Vegetation. Foram consideradas nas avalições os municípios com mais de 10.000 hectares de pastagens cultivadas. As análises indicaram que 173 municípios estão com mais de $50 \%$ de suas pastagens cultivadas sob algum processo de degradação. Em geral, conclui-se que o uso de séries temporais de NDVI constitui em importante parâmetro biofísico para aplicação de metodologias de avaliação das condições das pastagens do bioma Cerrado e corroboram para análises de inteligência territorial estratégica voltadas para o planejamento e a implementação de ações público-privadas de recuperação do potencial produtivo das áreas de pastagens em escala municipal.
\end{abstract}

Palavras chave: pecuária, planejamento rural, políticas públicas, sensoriamento remoto.

\section{ASSESSMENT OF THE PASTURES CONDITIONS IN THE BRAZILIAN SAVANNA BY MEANS GEOTECHNOLOGIES}

\begin{abstract}
The Brazilian Savanna covers 203.4 million hectares (24\% of the country territory), and about 55 million hectares are cultivated with pastures. This study aimed to evaluate cultivated pastures conditions in Brazilian Savanna. Geoprocessing techniques and Normalized Difference Vegetation Index (NDVI) timeseries data, derived from Spot-Vegetation sensor were applied. Municipalities with more than 10,000 hectares of cultivated pastures were considered. Analyzes based on NDVI time-series data indicated that 173 municipalities have more than 50\% of their cultivated pastures under some degradation process. In general, NDVI data were relevant to methodologic application to evaluate the cultivated pastures conditions of the Cerrado biome and corroborates to strategic territorial intelligence analyzes aimed at the planning and implementation of public and private actions to pastures productive potential recovery at a municipal scale.
\end{abstract}

Keywords: livestock, rural planning, public policies, remote sensing.

\section{INTRODUÇÃO}

No Brasil, a dinâmica de uso e cobertura da terra possui características próprias e diferenciadas em cada um dos biomas. No bioma Cerrado, cerca de $39 \%$ da vegetação natural passou por alterações, com destaque para a porção sul da região com apenas $15 \%$ de vegetação nativa (Sano et al., 2010). Nos últimos anos, o agronegócio brasileiro tem se desenvolvido consideravelmente em áreas de Cerrado que, atualmente, responde por cerca de $60 \%$ da produção de grãos do País. Além disso, estima-se que essa região possui mais de $30 \%$ do rebanho brasileiro, sendo pujante em termos de produção de carne e leite (Batistella et al., 2011). No entanto, apesar do bioma ocupar $24 \%$ do território nacional e gerar a maior produção agropecuária do País, muitas áreas, especialmente no âmbito daquelas com pastagens cultivadas (55 milhões de hectares), podem estar em processo de degradação ou degradadas (Peron \& Evangelista, 2004; Andrade et al., 2016).

${ }^{1}$ Pesquisadores na Empresa Brasileira de Pesquisa Agropecuária (EMBRAPA) 
O uso sustentável das pastagens é um tema estratégico para o País (Andrade et al., 2013), haja vista que a recuperação do potencial produtivo dessas terras, por exemplo, pode auxiliar na queda ou manutenção de baixos custos na produção de carne e leite, além de colaborar para a redução das emissões de gases de efeito estufa (GEEs). Essa temática se torna ainda mais relevante com a aprovação do Acordo de Paris (Brasil, 2015a). No âmbito desse Acordo, a contribuição do Brasil considera iniciativas para três setores (mudança do uso da terra e florestas, energia e agropecuária) que representam a maior participação no perfil brasileiro de emissões em 2012. Dentre essas iniciativas, destaque para o fortalecimento do Plano ABC (Agricultura de Baixo Carbono) como a principal estratégia para o desenvolvimento sustentável na agricultura, inclusive por meio da restauração de 15 milhões de hectares de pastagens degradadas até 2030 (Brasil, 2015b).

Os motivos que levam uma pastagem a passar por sucessivos estágios de degradação são geralmente bem conhecidos (Macedo, 2009; Dias-Filho, 2011), embora possam variar de acordo com as características edáficas e climáticas locais (Pedreira et al., 1998; Nascimento Junior, 1998). A recuperação do potencial produtivo de áreas com pastagens degradadas minimiza a pressão pela abertura de novas fronteiras agrícolas e pecuárias em detrimento de áreas com cobertura vegetal nativa. Em pastos recuperados é possível alcançar maior produtividade, mitigar as emissões de GEEs por meio do sequestro do dióxido de carbono $\left(\mathrm{CO}_{2}\right)$ atmosférico e, como consequência, prestar importante serviço ambiental ao conservar esse carbono no solo. Assim, a pecuária pode ser uma atividade economicamente mais rentável e ambientalmente mais eficiente (Andrade et al., 2016). Por outro lado, pastos mal manejados podem tornar-se emissores potenciais de GEEs (Dias-Filho, 2011), sendo de fundamental importância a avaliação e escolha com cautela das práticas de manejo adotadas ao levar em consideração questões como ajustes na taxa de lotação, uso de irrigação suplementar e de corretivos e fertilizantes, dentre outras que podem influenciar nas taxas de emissões de GEEs por animal.

A identificação e o mapeamento avaliativo das condições das pastagens em escalas municipais, regionais e nacionais são etapas fundamentais para a formulação e o fortalecimento de políticas de apoio, bem como para a avaliação do efetivo cumprimento das metas acordadas. O que possibilita dimensionar de forma ampla e eficiente a magnitude do problema e com isso contribuir para o planejamento de ações corretivas e tomadas de decisões do pecuarista sobre a recuperação, o manejo e ou uso dessas terras (Milne et al., 2007). Nesse contexto, procedimentos inovadores baseados em geotecnologias, como imagens de satélites, sistemas de posicionamento global (GPS) e sistemas de informações geográficas (SIG), podem auxiliar no desenvolvimento e no direcionamento de políticas públicas e diretrizes com visão de futuro.

Dessa forma, o presente estudo objetivou avaliar as condições das pastagens cultivadas em municípios localizados no bioma Cerrado por meio de séries temporais de índices de vegetação por diferença normalizada (NDVI) gerada a partir de imagens do sensor Spot-Vegetation.

\section{MATERIAL E MÉTODOS}

A área de estudo compreende as pastagens cultivadas do bioma Cerrado (Sano et al., 2008). Para as avaliações das condições das pastagens foram considerados os municípios que possuíam acima de 10.000 hectares de pastagens cultivadas, totalizando 806 municípios, abrangendo 13 estados: Bahia (BA), Distrito Federal (DF), Goiás (GO), Maranhão (MA), Mato Grosso (MT), Mato Grosso do Sul (MS), Minas Gerais (MG), Pará (PA), Paraná (PR), Piauí (PI), Rondônia (RO), São Paulo (SP), Tocantins (TO).

Após a definição territorial de estudo, utilizouse do produto NDVI (síntese de 10 dias) provenientes de imagens do sensor Spot Vegetation (VGT-S10). As imagens foram obtidas, para o período de janeiro de 2006 a setembro de 2011, na base de dados disponibilizada pelo Flemish Institute for Technological Research (Vlaamse Instelling Voor Technologisch Onderzoek - VITO NV, http://www.vito-eodata.be/PDF/portal/ Application.html\#Home). Os dados originais de NDVI (1 km de resolução espacial) são escalonados em valores 0 a 255 (níveis de cinza). Para converter os valores de NDVI no intervalo que varia de $-1 \mathrm{a}+1$, aplicou-se a equação descrita abaixo, proposta por Liu et al. (2010):

$$
\text { NDVI }=(\text { DN x 0,004) }-0,1
$$

Em que, NDVI é o índice de vegetação por diferença normalizada e DN é o número digital de cada pixel da imagem. Esta conversão é necessária para trabalhar os valores de NDVI como uma grandeza física e assim poder comparar os dados entre si ao longo da série temporal. 
O Slope foi utilizado para avaliar a mudança do NDVI ao longo da série como um todo. A partir da série temporal de dados NDVI a análise de regressão linear foi utilizada para simular a tendência de alterações positivas ou negativas em áreas de pastagens. Sendo Slope o coeficiente de inclinação da linha de regressão ajustada em cada pixel. Se Slope for positivo, indica que a vegetação pode estar em processo de recuperação, enquanto que valores negativos de Slope pode indicar a ocorrência de algum processo de degradação (Liu et al., 2010).

$$
\text { Slope }=\frac{\sum_{i=1}^{n}\left(Y_{i}-\bar{Y}\right)\left(Y_{N D V I_{i}}-\overline{Y_{N D V I}}\right)}{\sum_{i=1}^{n}\left(Y_{i}-\bar{Y}\right)^{2}}
$$

Em que, n é igual a 6 devido a utilização de uma série de 6 anos de dados NDVI (período de 2006 a 2011); i representa o ano 1 para 2006, ano 2 para 2007 até o ano 6 para 2011; $Y_{N D V I_{i}}$ é o valor máximo do NDVI no ano 1.

Neste estudo, para cada município, foram analisadas as áreas de pastagens cultivadas no bioma Cerrado conforme cenário condicional estipulado por Andrade et al. (2016). Nesse caso, há indicativos de degradação das pastagens quando Slope for menor que 0,001, ou seja, considera também como pastagem degradada a condição de estabilidade da vegetação $(-0,001$ a 0,001$)$ ao supor que tal estabilização ocorre dentro de algum estágio de degradação.

Posteriormente, os dados gerados em formato "raster" referente aos resultados da identificação das áreas de pastagens cultivadas com e sem indicativos de degradação, foram transformados para a formato "vetorial". Tal transformação possibilitou aplicar ferramentas de geoprocessamento para quantificar as áreas de pastagens com e sem indicativos de degradação, bem como a área de cada município que possui mais de 10.000 hectares de pastagens cultivadas, no bioma Cerrado. Em seguida, foram gerados os dados de porcentagem de pastagem cultivada em relação à área total do município, porcentagem de área de pastagem cultivada degradada em relação à área total do município e porcentagem de área com pastagens cultivadas que apresentam indicativos de degradação em relação à área total de pastagem cultivada em cada município.
Esses resultados foram gerados no formato de "tabela de atributos" no software ArcGis versão 10.1. Na sequência, a partir dos resultados apresentados na tabela de atributos, foram elaborados os mapas para análise avaliativa das condições das pastagens cultivadas em escala municipal.

\section{RESULTADOS E DISCUSSÃO}

A porcentagem do total de área de pastagem cultivada em relação à área municipal pode ser visualizada na Figura 1 e a porcentagem de área de pastagem cultivada degradada em relação à área total do município pode ser visualizada na Figura 2. Podese observar que muitos municípios estão representados em porcentagens de classes em tons de verde (Figuras 1 e 2), com abrangência principalmente na maior parte dos municípios do Mato Grosso, Pantanal e Matopiba (Maranhão, Tocantis, Piauí e Bahia). E algumas justificativas podem estar no fato que esses municípios de Mato Grosso possuem maior área territorial e parte significativa de seu território é destinada a outros usos como de culturas de ciclo curto (soja, milho, algodão e outras) (Brown et al., 2013). Já na região do Pantanal, predominam pastagens naturais em que boa parte são utilizadas para cria e recria do gado (Santos et al., 2002), sendo essas pastagens não avaliadas no presente estudo. No que se refere à região do Matopiba, uma das explicações pode estar no fato dos municípios terem economia mais fundamentada na produção de grãos e fibras e, consequentemente, de maior uso do território (Brasil, 2015c).

Nota-se na Figura 2 que os municípios das regiões oeste, centro, noroeste e norte de Goiás se destacaram por apresentar indicativos de degradação de pastagens em mais de $35 \%$ da área total do município, conforme representado nas classes em tons de laranja a vermelho. Ressalta-se também as classes que variam de $8 \%$ (amarelo) a $25 \%$ (laranja claro) e que abrange boa parte dos municípios de Goiás, Minas Gerais, Mato Grosso e Mato Grosso do Sul.

Ao fazer uma análise geral entre os mapas das Figuras 1 e 2 observa-se que há maior porcentagem de indicativos de degradação em municípios que possuem mais pastagens cultivadas em seu território. Destaque para os municípios da região central de Goiás que possuem mais de $50 \%$ do seu território com pastagens cultivadas. Além disso, é notório porcentagens acima de $40 \%$ em regiões Sul e Noroeste de Goiás, na maior 


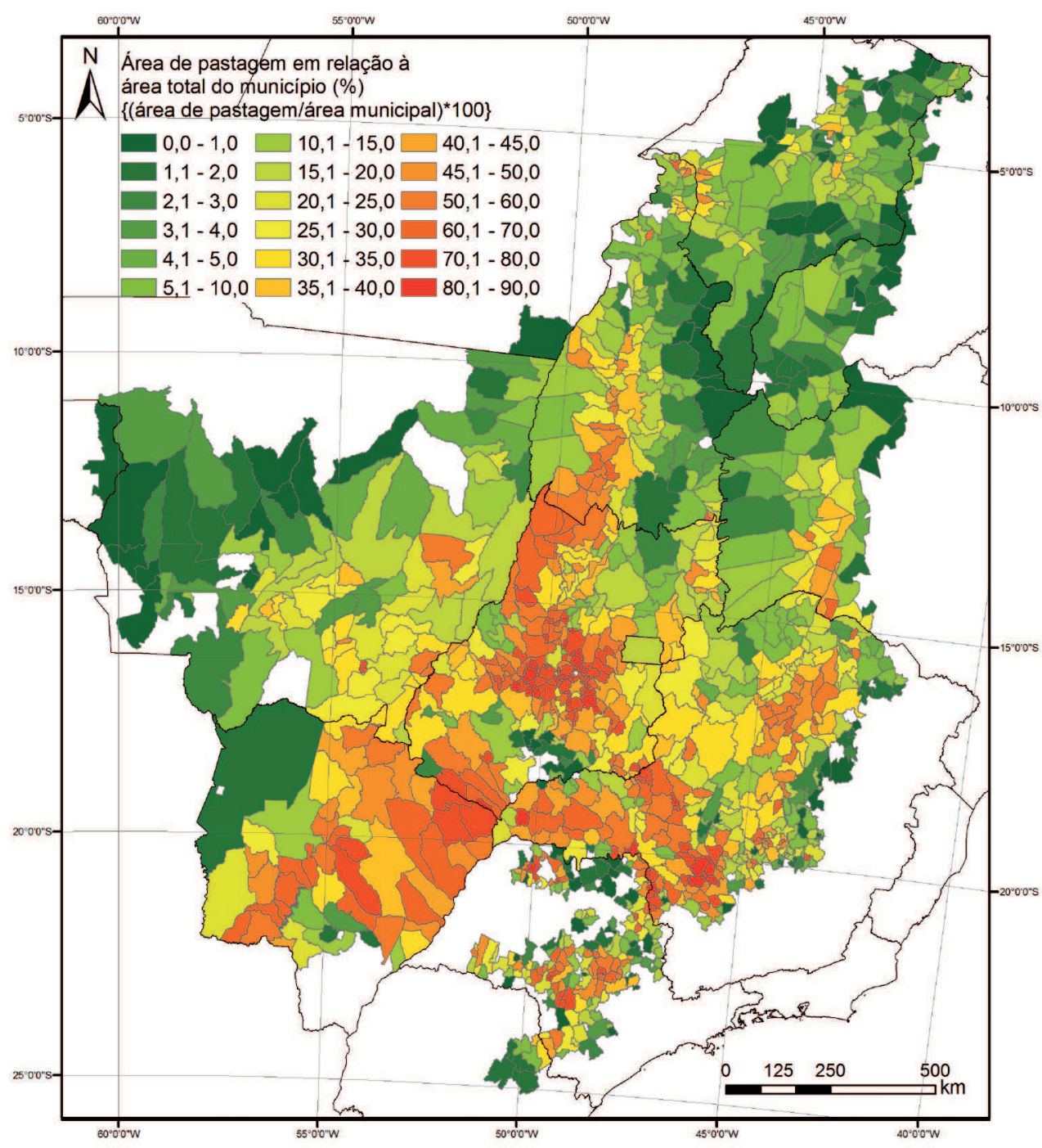

Figura 1. Mapa de porcentagem de área de pastagem cultivada em relação à área total do município, no bioma Cerrado.

parte dos municípios de Mato Grosso do Sul, Triângulo Mineiro, Alto Paranaíba, Centro Oeste de Minas e em parte dos municípios do Norte de Minas Gerais. Nestas regiões, há indicativos de degradação em mais de $10 \%$ das áreas de pastagens cultivadas, podendo chegar a valores superiores a $30 \%$ em alguns dos municípios.

Visualiza-se no mapa ilustrado na Figura 3 a porcentagem de área de pastagens com indicativos de degradação em relação à área total de pastagem cultivada em cada município. Há indicativos de degradação em mais de a 50\% (tons em vermelho) da área total de pastagens cultivadas em vários municípios das regiões oeste, centro, noroeste e norte de Goiás, nordeste mato-grossense, sudoeste do Mato Grosso do Sul e alguns municípios da região do Matopiba. Além disso, porcentagens acima de 50\% também foram observadas para alguns municípios de Minas Gerais e São Paulo, no entanto, de forma menos concentrada por região. Em um total de 806 municípios avaliados, identificou-se que 173 apresentaram indicativos de degradação em mais de $50 \%$ das áreas de pastagens cultivadas. Vale ressaltar também que classes de 


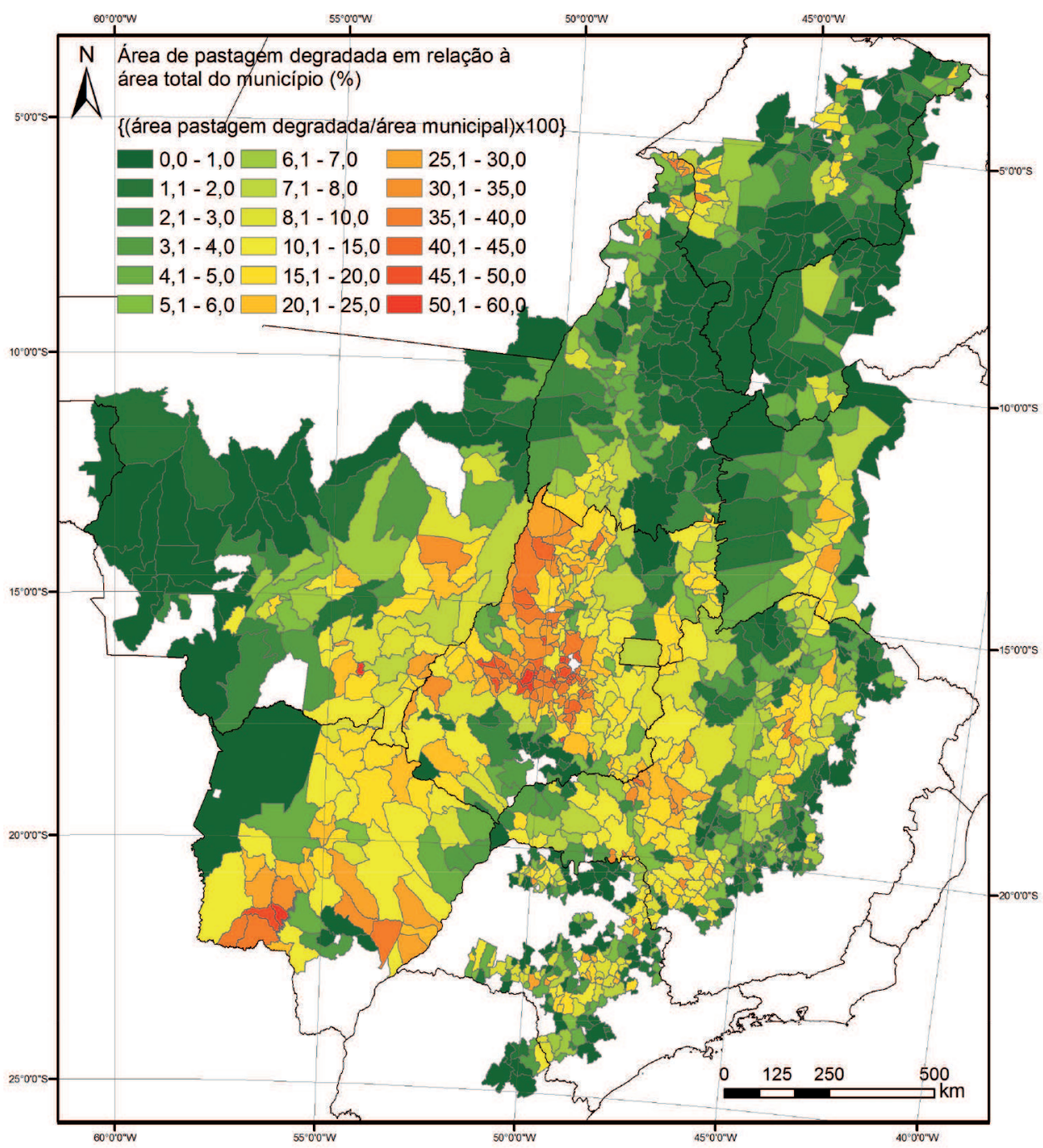

Figura 2. Mapa da porcentagem de área com pastagem cultivada que apresenta indicativos de degradação em relação à área total do município, no bioma Cerrado.

indicativos de degradação entre 20 e $40 \%$ das pastagens cultivadas por município foram observadas de forma dispersa no bioma Cerrado. Já as classes em tons de verde (menos de $10 \%$ de indicativos de degradação) se concentraram na porção oeste de Mato Grosso e Mato Grosso do Sul e em municípios da região do Matopiba.

Em geral, as elevadas porcentagens de áreas de pastagens cultivadas com indicativos de degradação podem ter como uma das justificativas os indícios de tendência de queda nos índices de produtividade, sugerindo que os sistemas de produção utilizados não têm possibilitado produtividades sustentáveis (Macedo, 1995). Assim, o contínuo empobrecimento dos solos das áreas de pastagens tem levado a um processo de degradação em que há substituição de espécies introduzidas (Brachiaria, por exemplo) por espécies menos exigentes em fertilidade do solo e manejo do pastejo, como a grama mato grosso e até mesmo barba de bode, numa etapa mais avançada de degradação (Moraes et al., 1995). Vale lembrar que, nem sempre a presença de invasoras é um sinal de declínio da fertilidade 


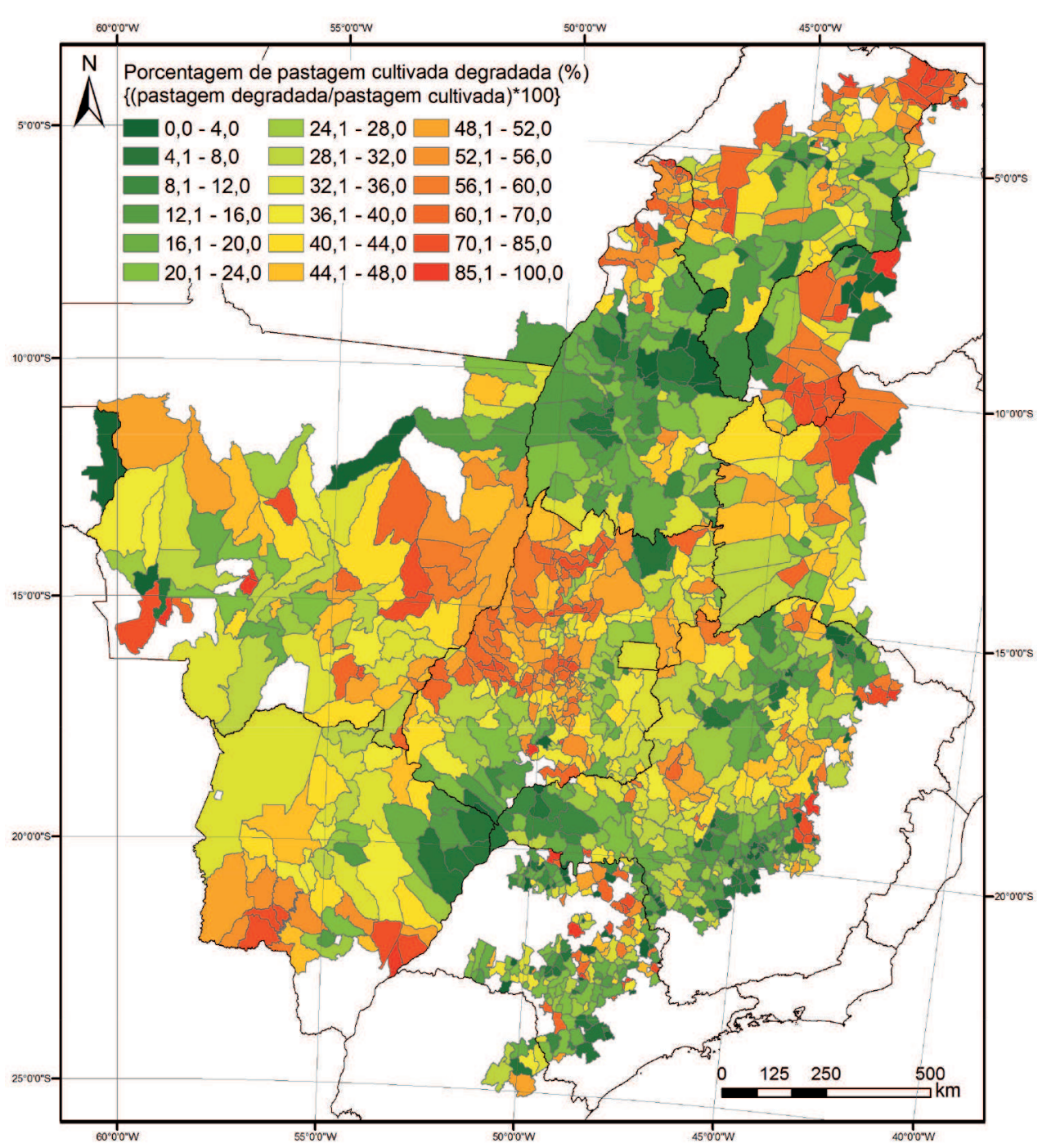

Figura 3. Mapa de porcentagem de área com pastagens cultivadas que apresentam indicativos de degradação em relação à área total de pastagem cultivada em cada município, no bioma Cerrado.

do solo, sendo que algumas delas podem ser favorecidas pela adubação ou pela condição de melhor fertilidade do solo, neste caso, seria mais um reflexo do mau manejo, como por exemplo, o subpastejo (Dias-Filho, 1990).

Outro ponto importante é que o adequado manejo do pastejo deve ser entendido como a possibilidade de se alterar as taxas de lotação de modo a sincronizálas com a capacidade de suporte das pastagens, proporcionando maior produtividade, sem comprometimento de sua persistência. O superpastejo, causado por altas taxas de lotação, por promover decréscimos na quantidade de forragem produzida, com consequentes decréscimos na produção animal, é um dos fatores que contribuem para a degradação das pastagens (Zimmer \& Corrêa, 1993; Euclides, 1994).

\section{CONCLUSÃO}

As análises baseadas em séries temporais de NDVI, indicaram que 173 municípios do Cerrado brasileiro estão com mais de $50 \%$ de suas pastagens cultivadas sob algum processo de degradação. O uso de geotecnologias para a análise de parâmetros biofísicos 
das condições das pastagens permite compreender o espaço rural de forma multiescalar. As análises envolvendo inteligência territorial estratégica são fundamentais para o planejamento e a implementação de ações público-privadas de recuperação do potencial produtivo das áreas de pastagens em escala municipal.

\section{LITERATURA CITADA}

ANDRADE, R.G.; BOLFE, E.L.; VICTORIA, D.C.; NOGUEIRA, S.F. Geotecnologia - Recuperação de pastagens no Cerrado. Agroanalysis (FGV), v.36, p.30-33, 2016.

ANDRADE, R.G.; RODRIGUES, C.A.G.; SANCHES, I.D.; TORRESAN, F.E.; QUARTAROLI, C.F. Uso de técnicas de sensoriamento remoto na detecção de processos de degradação de pastagens.

Engenharia na Agricultura, v.21, p.234$243,2013$.

BATISTELLA, M.; ANDRADE, R.G.; BOLFE, E.L.; VICTORIA, D.C.; SILVA, G. B.S. Geotecnologias e gestão territorial da bovinocultura no Brasil.

Revista brasileira de zootecnia

(Online), v.40, S.E., p.251-260, 2011.

BRASIL. Ministério do Meio Ambiente. Acordo de Paris. 12 de dezembro de 2015. Versão em português. 13p. 2015a. Disponível em: <http:// www.mma.gov.br/clima/convencao-das-nacoesunidas/acordo-de-paris>. Acesso em: 28 out. 2016.

BRASIL. Ministério do Meio Ambiente.

Fundamentos para a elaboração da Pretendida Contribuição Nacionalmente Determinada (iNDC) do Brasil no contexto do Acordo de Paris sob a UNFCCC. 13p. 2015b. Disponível em: <http:// www.mma.gov.br/images/arquivos/clima/convencao/ indc/Bases_elaboracao_iNDC.pdf > . Acesso em: 28 out. 2016.

BRASIL. Portal Brasil. Economia e Emprego: Matopiba se consolida como nova fronteira agrícola do País. Agropecuária - São 324 mil estabelecimentos agrícolas no MA, TO, PI e BA, em área que produziu $9,4 \%$ da safra de grãos 2014/2015. 2015c. Disponível em: <http://www.brasil.gov.br/ economia-e-emprego/2015/10/matopiba-seconsolida-como-nova-fronteira-agricola-do-pais $>$. Acesso em: 29 mar. 2017.
BROWN, J.C.; KASTENS, J.H.; COUTINHO, A.C.; VICTORIA, D.C.; BISHOP, C. R. Classifying multiyear agricultural land use data from Mato Grosso using time-series MODIS vegetation index data. Remote Sensing of

Environment, v.130, p.39-50, 2013.

DIAS-FILHO, M.B. Degradação de pastagens: processos, causas e estratégias de recuperação. 4a. ed. rev., atual. e ampl. Belém, PA, 2011.

DIAS-FILHO, M.B. Plantas invasoras em pastagens cultivadas da Amazônia: estratégias de manejo e controle. Belém: Embrapa-CPATU, 1990. 103p. (Documentos, 52).

EUCLIDES, V.P.B. Algumas considerações sobre manejo das pastagens. Campo Grande: EMBRAPA-CNPGC, 1994. 31p. (Documentos, 57).

LIU, S.; WANG, T.; GUO, J.; QU, J.; AN, P. Vegetation change based on SPOT-VGT data from 1998-2007, northern China.

Environmental Earth Sciences, v.60, p.1459-1466, 2010.

MACEDO, M.C.M. Integração lavoura e pecuária: o estado da arte e inovações tecnológicas.

Revista Brasileira de Zootecnia, v.38, número especial, p.133-146, 2009.

MACEDO, M.C.M. Pastagens no ecossistema cerrado: pesquisa para o desenvolvimento sustentável. In: SIMPÓSIO SOBRE PASTAGENS NOS ECOSSISTEMAS BRASILEIROS: PESQUISAS PARA O DESENVOLVIMENTO SUSTENTÁ VEL, 32, Brasília, 1995. Anais... Brasília, Sociedade Brasileira de Zootecnia, 1995. p.28-62.

MILNE, E.; AL ADAMAT, R.; BATJES, N.H.; BERNOUX, M.; BHATTACHARYYA, T.; CERRI, C.C.; CERRI, C.E.P.; COLEMAN, K.; EASTER, M.; FALLOON, P.; FELLER, C.; GICHERU, P.; KAMONI, P.; KILLIAN, K.; PAL, D.K.;

PAUSTIAN, K.; POWLSON, D.S.; RAWAJûH, Z.; SESSAY, M.; WILLIAMS, S.; WOKABI, S.

National and sub-national assessments of soil organic carbon stocks and changes: The GEFSOC modelling system. Agriculture, Ecosystems and Environment. v.122, p. 3-12, 2007. 
MORAES, A.; MARASCHIN, G. E.; NABINGER, C. Pastagens nos ecossistemas de clima subtropical: Pesquisas para o desenvolvimento sustentável. In: SIMPÓSIO SOBRE PASTAGENS NOS ECOSSISTEMAS BRASILEIROS: PESQUISAS PARA O DESENVOLVIMENTO SUSTENTÁ VEL, 32, Brasília, 1995. Anais... Brasília, Sociedade Brasileira de Zootecnia, 1995. p.147-200.

NASCIMENTO JÚNIOR, D. Ecossistema de pastagem cultivadas. In: Anais do $15^{\circ}$ Simpósio sobre Manejo da Pastagem. FEALQ. p. 271-296, 1998.

PEDREIRA, C.G.S.; NUSSIO, L.G.; SILVA, S.C. Condições Edafo-climáticas para produção de Cynodon spp. In: Anais do $15^{\circ}$ Simpósio sobre Manejo da Pastagem. FEALQ. p.85-113, 1998.

PERON, A.J.; EVANGELISTA, A.R. Degradação de pastagens em regiões do cerrado. Ciência e Agrotecnologia, v.28, n.3, p.655-661, 2004.
SANO, E.E.; ROSA, R.; BRITO, J.L.S.; FERREIRA, L.G. Land cover mapping of the tropical savanna region in Brazil. Environmental Monitoring and Assessment, v.166, p.113-124, 2010.

SANO, E.E.; ROSA, R.; BRITO, J.L.S.; FERREIRA, L.G. Mapeamento semidetalhado do uso da terra do Bioma Cerrado. Pesquisa Agropecuária Brasileira, v.43, p.153-156, 2008.

SANTOS, S.A.; COSTA, C.; CRISPIM, S.M.A.; PELLEGRIN, L.A.; RAVAGLIA, E. Estimativa da capacidade de suporte das pastagens nativas do Pantanal, sub-região da Nhecolândia. Corumbá: Embrapa Pantanal, 2002. 31p. (Embrapa Pantanal. Boletim de Pesquisa e Desenvolvimento, 27). Disponível em: <http://www.cpap.embrapa.br/ publicacoes/online/BP27.pdf>. Acesso em: 29 mar. 2017.

ZIMMER, A.H.; CORREA, E.S. A pecuária nacional, uma pecuária de pasto? In: ENCONTRO SOBRE RECUPERAÇÃO DE PASTAGENS, 1., Nova Odessa, 1993. Anais... Nova Odessa: Instituto de Zootecnia, 1993. p.1-25.

Recebido para publicação em 30/9/2016 e aprovado em 19/3/2017. 